\title{
Gamma knife radiosurgery for recurrent gliomas
}

\author{
Zjiwar H. A. Sadik ${ }^{1,2,3}$. Patrick E. J. Hanssens ${ }^{1}$. Jeroen B. Verheul ${ }^{1,2} \cdot$ Guus N. Beute $^{1,2} \cdot$ Suan Te Lie ${ }^{1,2}$. \\ Sieger Leenstra ${ }^{4} \cdot$ Hilko Ardon ${ }^{1,2}$
}

Received: 20 June 2018 / Accepted: 21 August 2018 / Published online: 6 September 2018

(c) The Author(s) 2018

\begin{abstract}
Objective In recent years, gamma knife radiosurgery (GKRS) has become increasingly more popular as a salvage treatment modality for patients diagnosed with recurrent gliomas. The goal of GKRS for recurrent glioma patients is to improve survival rates with minimal burden for these patients. The emphasis of this report is on local tumor control (TC), clinical outcome and survival analysis.

Methods We performed a retrospective analysis of prospectively collected data of all patients who underwent GKRS for gliomas at the Gamma Knife Center Tilburg between 23-09-2002 and 21-05-2015. In total, 94 patients with glioma were treated with GKRS. Two patients were excluded because GKRS was used as a first stage treatment. The other 92 patients were included for analysis.

Results TC was 37\% for all tumors (TC was 50\% in LGGs and 27\% in HGGs). Local progression (LP) was $46 \%$ for all tumors (LP was 31\% in LGGs and 58\% in HGGs). New distant lesions were seen in 18\% of all patients (in 5\% of LGG patients and $31 \%$ of HGG patients). Median progression-free and overall survival (PFS and OS) for all patients were 10.5 and 34.4 months, respectively. Median PFS was 50.1 and 5.7 months for low and high grade tumors, respectively. Median OS was 86.6 and 12.8 months for low and high grade tumors, respectively. No serious adverse events were noted post-GKRS.

Conclusion GKRS can safely be used as salvage treatment for recurrent glioma and seems to improve survival rates in (high grade) glioma patients with minimal burden.
\end{abstract}

Keywords Gamma knife radiosurgery $\cdot$ Recurrent $\cdot$ Gliomas

\section{Introduction}

Gliomas are the most common primary malignant brain tumors. The overall age-adjusted incidence rates for all gliomas range between $4.67-5.73 / 100,000$ persons/year [1,

Portions of this work were presented at the 18th Leksell Gamma Knife Society Meeting, Amsterdam, The Netherlands, May 15-19, 2016.

Zjiwar H. A. Sadik

s_shibie@hotmail.com

1 Gamma Knife Center, Elisabeth-Tweesteden Hospital, Tilburg, The Netherlands

2 Department of Neurosurgery, Elisabeth-Tweesteden Hospital, Tilburg, The Netherlands

3 Department of Neurosurgery, Amsterdam Medical Center, Amsterdam, The Netherlands

4 Department of Neurosurgery, Erasmus Medical Center, Rotterdam, The Netherlands
2]. Gliomas have been classified into four grades of ascending malignancy by the World Health Organization (WHO). Essentially, they can be divided into two major groups: low grade gliomas (LGG; WHO 1-2) and high grade gliomas (HGG; WHO 3-4). GBM (WHO grade 4) is the most deadly glioma in adults with an overall 5 year survival of $0.05-4.7 \%$ [3-7]. Generally, gliomas are more common in men than in women $[3,8-10]$. Currently, the treatment of gliomas consists of maximal safe surgical resection, external beam radiation therapy (EBRT), and chemotherapy. However, these tumors do often reoccur. Options for salvage treatment are repeated surgery, re-irradiation with EBRT, chemotherapy, novel therapies, or a combination of these treatments. Repeated surgery can be a good option as a salvage treatment, but might be associated with postoperative complications. Treatment with EBRT for a second time can be accompanied by high risk of radiation-related toxicity and necrosis. In recent years, gamma knife radiosurgery (GKRS) has become increasingly more popular as a salvage treatment 
modality for patients diagnosed with recurrent gliomas. The goal of GKRS for recurrent glioma patients is to improve survival rates with minimal burden for these patients. We summarize our experience in a group of 92 patients with recurrent glioma, treated with GKRS. The emphasis of this report is on local tumor control (TC), clinical outcome and survival analysis.

\section{Methods}

\section{Case selection}

We performed a retrospective analysis of prospectively collected data of all patients who underwent GKRS for gliomas at the Gamma Knife Center Tilburg between 23-09-2002 and 21-05-2015. The study was approved by the medical ethical committee. Hospital records, including clinical notes, doctors' letters, radiology reports and demographic data, were reviewed and relevant information was extracted for analysis. Pre- and post-GKRS clinical characteristics were reviewed. We included all patients with histologically confirmed gliomas. In total, 94 patients with glioma were treated with GKRS. Two patients were excluded because GKRS was used as a first stage treatment. The other 92 patients were included for analysis.

\section{Baseline characteristics}

As stated, 92 patients (52 males and 40 females) underwent GKRS for recurrent glioma. The median age at time of GKRS was 50 years (range 7-76) (Table 1). The histopathology of the tumors is summarized in Table 2. Eighty-five patients had undergone at least one operation before GKRS. Seven patients received biopsy followed by adjuvant treatment in the form of chemotherapy, EBRT or a combination of both, before GKRS. Pre-GKRS treatment features are summarized in Table 1.

\section{Gamma knife radiosurgical procedure}

The GKRS procedure was performed using the Leksell Gamma Knife 4C, before 2009, and Leksell Gamma Knife Perfexion thereafter. GammaPlan Software (Elektra) was used for treatment planning. The application of the Leksell G-Frame with fixation posts and screws at four points was performed at the patients room using a local anaesthetic solution ( $9 \mathrm{ml}$ of lidocaine $2 \%$ + epinephrine $0.125 \%$ combined with $1 \mathrm{ml}$ of $\mathrm{NaHCO}_{3} 84 \mathrm{~g} / 1000 \mathrm{ml}$ ). Following frame placement, high resolution stereotactic MRI was performed for treatment planning. Pre- and post-contrast (Triple dose Gadolinium) T1 weighted axial images were obtained with a slice thickness of $1.5 \mathrm{~mm}$. Stereotactic radiosurgery and
Table 1 Pre-GKRS patient characteristics and radiosurgical features

\begin{tabular}{ll}
\hline Patient characteristics & No. of patients $(\%)$ \\
\hline Total patients included & 92 \\
Male: female ratio & $52: 40$ \\
Median age (range) & $50(7-76)$ \\
Median Karnofski index (range) & $90(50-100)$ \\
$\geq 70$ & 80 \\
$<70$ & 12 \\
Histopathology & \\
WHO I glioma & $17(19 \%)$ \\
WHO II glioma & $26(28 \%)$ \\
WHO III glioma & $24(26 \%)$ \\
WHO IV glioma & $25(27 \%)$ \\
Primary surgery & \\
Complete resection & $78(84 \%)$ \\
Subtotal resection & $7(8 \%)$ \\
Biopsy & $7(8 \%)$ \\
Adjuvant therapy & \\
Repeated surgery & $1(1 \%)$ \\
PVC + EBRT + adjuvant TMZ & $26(28 \%)$ \\
EBRT + TMZ & $2(2 \%)$ \\
EBRT only & $40(43 \%)$ \\
Target location & \\
Infratentorial & $19(18 \%)$ \\
Supratentorial & $88(82 \%)$ \\
Unifocal & $79(86 \%)$ \\
Multifocal & $13(14 \%)$ \\
Redian tumor volume in cm ${ }^{3}$ (range) & $2.22(0.01-9.95)$ \\
Median PD in Gy (range) & \\
Median minimal dose in Gy (range) & $18(12-25)$ \\
Median maximal dose in Gy (range) & \\
Median isodose in \% (range) & \\
Median coverage in \% (range) & \\
Median no. of lesions treated (range) & \\
\hline
\end{tabular}

$E B R T$ External beam radiotherapy, $P D$ Prescribed dose, $P V C$ Procarbazine, Lomustine and vincristine, TMZ Temozolomide

dose planning were then performed in consultation with a neurosurgeon, radiation-oncologist, and medical physicist. The target was defined as the contrast enhancing lesion on the planning MRI-scan. Target delineation was limited to the target enhancing lesion only for progressive LGG (WHO I and II) and HGG (WHO III and WHO IV) as confirmed by the ASTRO guidelines [11]. The median number of lesions treated with GKRS was 1 (range: $1-4$ ) with a median tumor volume of $2.22 \mathrm{~cm}^{3}$ (range: 0.01-9.95). Eventually, a total of 107 (48 LGG and 59 HGG) tumors were treated with GKRS. The prescribed dose (PD) ranged between 12 and $25 \mathrm{~Gy}$ (median $18 \mathrm{~Gy}$ ) to that isodose covering $89-100 \%$ of the target (Table 1). 
Table 2 Histopathology and radiosurgical response according to the Tilburg radiological classification

\begin{tabular}{|c|c|c|c|c|c|c|}
\hline Histopathology & No. of patients & No. of tumors & $\mathrm{TC}$ & LP & MP & NDL \\
\hline \multicolumn{7}{|l|}{ WHO I } \\
\hline Pilocytic astrocytoma & 10 & 10 & 7 & 3 & & \\
\hline Subependymoma & 4 & 4 & 2 & 1 & 1 & \\
\hline Myxopapillary ependymoma & 1 & 2 & 2 & & & \\
\hline Ganglioglioma & 1 & 1 & 1 & & & \\
\hline Subependymal giant cell astrocytoma & 1 & 1 & 1 & & & \\
\hline \multicolumn{7}{|l|}{ WHO II } \\
\hline Oligodendroglioma & 11 & 12 & 3 & 9 & & 5 \\
\hline Ependymoma & 10 & 13 & 11 & & 1 & 7+1 Lepto \\
\hline Astrocytoma & 2 & 2 & 1 & 1 & & \\
\hline Oligoastrocytoma & 2 & 2 & & 1 & 1 & 1 \\
\hline Central neurocytoma & 1 & 1 & 1 & & & 1 \\
\hline \multicolumn{7}{|l|}{ WHO III } \\
\hline Anaplastic oligodendroglioma & 11 & 15 & 4 & 6 & 3 & 5 \\
\hline Anaplastic astrocytoma & 7 & 10 & 4 & 3 & 2 & 4 \\
\hline Anaplastic ependymoma & 3 & 3 & 1 & 2 & & \\
\hline Anaplastic oligoastrocytoma & 3 & 3 & & 2 & 1 & 1 \\
\hline \multicolumn{7}{|l|}{ WHO IV } \\
\hline Glioblastoma multiforme & 25 & 28 & 7 & 21 & 1 & 5 \\
\hline Total & 92 & 107 & & & & \\
\hline
\end{tabular}

Lepto Leptomeningeal, LP Local progression, MP Marginal progression, NDL New distant lesion, TC Tumor control
The median PD for LGG and HGG was 18 Gy ranging between 12 and 25 Gy and 14-25 Gy, respectively. After GKRS, patients received $8 \mathrm{mg}$ dexamethasone for 5 days and thereafter the dose was decreased to $0 \mathrm{mg}$ within 1 week.

\section{Radiological classification for progression}

Determining radiological progression (after treatment) is a difficult task for gliomas, especially differentiating between progression/recurrence and treatment induced radiological changes. Therefore, we have introduced the Tilburg radiological classification for scoring TC after GKRS. Figure 1 shows the Tilburg classification on which we based our definitions of tumor progression. A subdivision was made between local TC with or without (a) new distant lesion(s).

\section{Statistical analysis}

Data are represented as median with the range. The Pearson correlation coefficient was obtained through linear regression analysis. Survival analysis was done by $\log$ rank test on Kaplan Meier survival estimates. Survival was defined as time from GKRS till death or last FU. Statistics were calculated with Prism software 7 (GraphPad Software Inc., San Diego, CA, USA).

\section{Results}

\section{Radiosurgical response/radiological tumor control}

TC was achieved in $37 \%$ of all tumors treated. According to tumor grade, TC was obtained in 50\% of LGGs (24 out of 48 tumors) and in $27 \%$ of HGGs (16 out of 59 tumors). Local progression (LP) occurred in $46 \%$ of all tumors, in $31 \%$ of LGGs (15 out of 48 tumors) and in 58\% of HGGs (34 out of 59 tumors). Marginal progression occurred in $9 \%$ of all tumors, in $6 \%$ of LGGs (3 out of 48 tumors) and in 12\% of HGGs (7 out of 59 tumors). New distant lesions (NDL) were seen in $18 \%$ of all patients; in 5\% of LGG patients (2 out of 43 patients) and $31 \%$ of HGG patients ( 15 out of 49 patients) (Table 2). One (LGG) patient had leptomeningeal disease (Table 2).

\section{Survival and prognostic factors/clinical outcome}

Kaplan Meier survival curves are given in Fig. 2. Median progression-free and overall survival (PFS and OS) for all patients were 10.5 and 34.4 months, respectively (Fig. 2). Median PFS was not yet reached for grade 1 (range 7.7-139.4 months), 14.3 months for grade 2 (range 2.8-139.1 months), 8.8 months for grade 3 (range 1.5-49.6 months) and 4.4 months for grade 4 glioma (range 0.4-18.1 months). Median OS for the 


\section{Classification with no distant lesion(s) during follow-up}

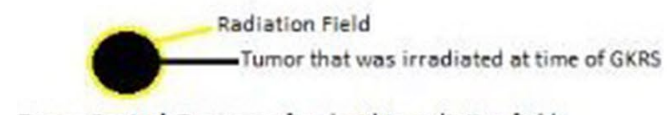

Tumor Control: Tumor confined within radiation fleld

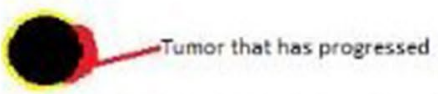

Marginal Progression: Progression in the edge of the tumor, outside the radiation field, on a specific location

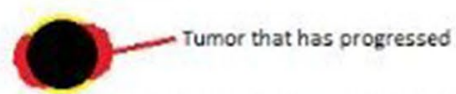

Marginal Progression: Progression in the edge of the tumor, outside the radiation field, on 2 specific locations

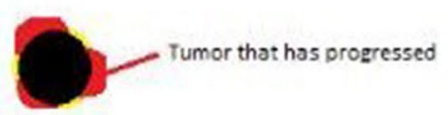

Local Progression: Progression in the edge of the tumor, outside the radiation field, on $>2$ locations

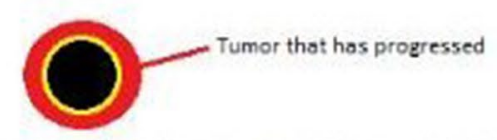

Local Progression: Progression of the tumor, outside the radiation field, in all directions
Classification with new distant lesion(s) during follow-up

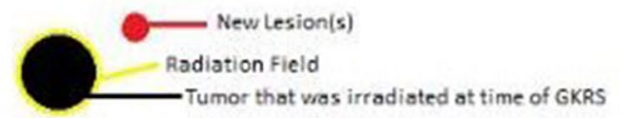

Tumor Control + New distant lesion(s): Tumor confined within radiation field, but new distant lesion(s) visible on follow-up MRI. This can be 21 lesion(s) in the ipsilateral or contraloteral hemisphere

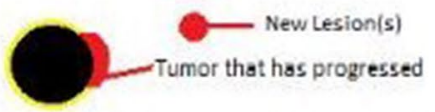

Marginal Progression + New distant lesion(s): Progression in the edge of the tumor, outside the radiation field, on a specific location with new distant lesion(s) visible on followrup MRI. This can be $\geq 1$ lesion(s) in the ipsilateral or contralateral hemisphere

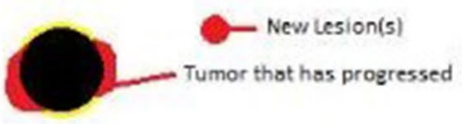

Marginal Progression + New distant lesion(s): Progression in the edge of the tumor, outside the radiation field, on 2 specific locations with new distant lesion(s) visible on the follow-up MRI. This can be $\geq 1$ lesion(s) in the ipsilateral or contralateral hemisphere

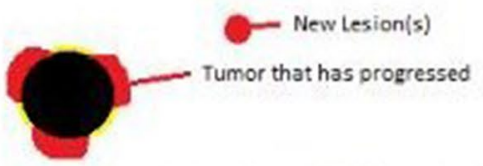

Local Progression + New distant lesion(s): Progression in the edge of the tumor, outside the radiation field, on $>2$ locations with new distant lesion(s) visible of the follow-up MRI. This can be 21 lesion(s) in the ipsilateral or contralateral hemisphere

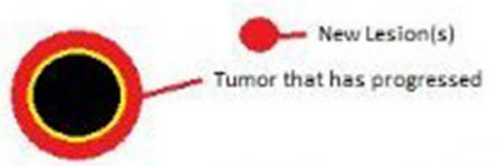

Local Progression + New distant lesion(s): Progression of the tumor, outside the radiation field, in all directions with new distant lesion(s) visible on the follow-up MRI. This can be $\geq 1$ lesion(s) in the ipsilateral or controlateral hemisphere

Fig. 1 Tilburg radiological classification

aforementioned tumor grades was not yet reached (range 7.7-139.4 months), 47.2 months (range 2.8-139.1 months), 18.1 months (range 3.3-52.0 months) and 10.4 months (range 1.4-32.7 months), respectively. There was a highly significant increase in both PFS and OS according to tumor grade; i.e. the lower the tumor grade, the better the outcome (Fig. 2). Low grade tumors were defined as grade 1 and 2 tumors, high grade tumors as grade 3 and 4 tumors. Median PFS was 50.1 months (range 1.3-139.4 months) and 5.7 months (range 0.4-92.5 months) for low and high grade tumors, respectively. Median OS was 86.6 months (range 1.3-139.4 months) and 12.8 months (range 0.5-109.5 months) for low and high grade tumors, respectively (Fig. 2). There was no correlation between time from first diagnosis to GKRS and PFS. The median followup (FU) was 34 months from the date of GKRS treatment.
We lost 56 patients during FU. All of these patients died due to intracranial progressive disease.

\section{Adverse events}

Seventy-nine percent (36 out of 48) of the LGG patients had no adverse events. Frame related swelling occurred in $10 \%$ ( 5 out of 48 ) of the patients within a median time of 14 days (Table 3 ).

In HGG patients, $59 \%$ had no adverse events. In this group the incidence of frame related swelling was comparable to that of LGG patients. Other adverse events for both LGG and HGG patients are summarized in Table 3. No serious adverse events were noted after GKRS (Table 3). 
PFS (all patients)

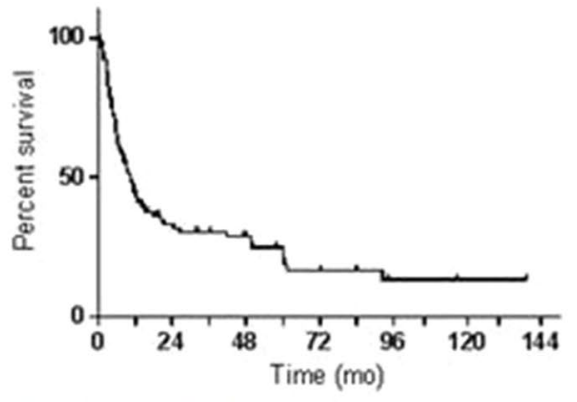

No. At Risk $92 \quad 23 \quad 16 \quad 8 \quad 3 \quad 2 \quad 0$

PFS (low grade vs high grade)

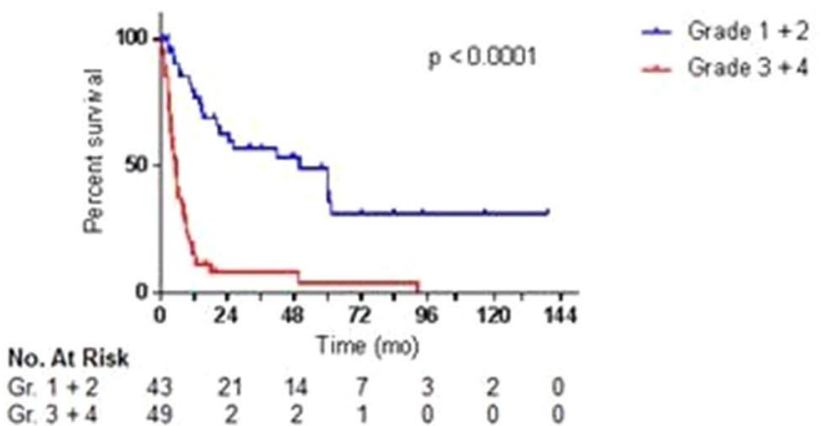

Fig. 2 Progression-free and overall survival (PFS and OS) for all patients and according to tumor grade (LGG vs. HGG). Median PFS was 10.5 months (range 0.4-139.4 months) and median OS was 34.4 months (range 0.5-139.4 months). (mo months). Low grade tumors were defined as grade 1 and 2 tumors, high grade tumors
OS (all patients)

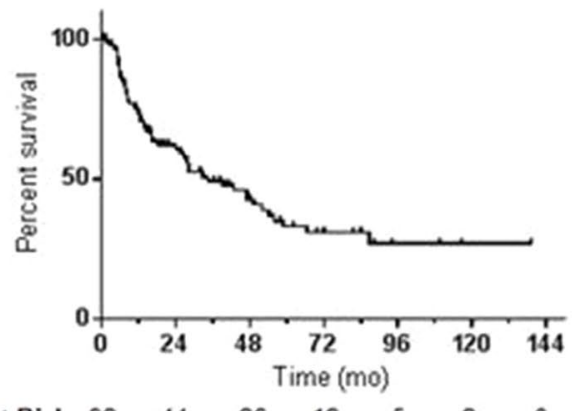

No. At Risk $92 \quad 44 \quad 26 \quad 12 \quad 5 \quad 2 \quad 0$

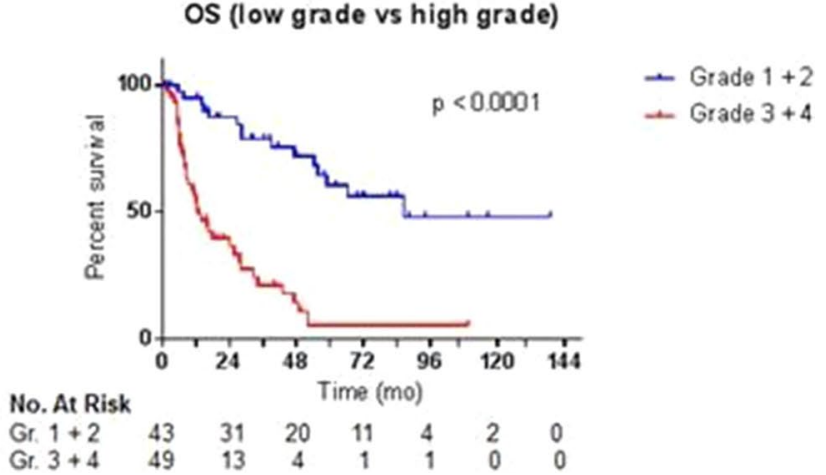

as grade 3 and 4 tumors. Median PFS was 50.1 months (range 1.3139.4 months) and 5.7 months (range 0.4-92.5 months) for low and high grade tumors respectively. Median OS was 86.6 months (range 1.3-139.4 months) and 12.8 months (range 0.5-109.5 months) for low and high grade tumors respectively. (mo months, $v s$ versus)
Table 3 Adverse events after radiosurgery

\begin{tabular}{lllll}
\hline Adverse events $^{\mathrm{a}}$ & LGG & Median time to occur & HGG & $\begin{array}{l}\text { Median } \\
\text { time to } \\
\text { occur }\end{array}$ \\
\hline None & $36(79 \%)$ & & $35(59 \%)$ & \\
Frame related swelling/pain & $5(10 \%)$ & 14 days & $5(9 \%)$ & 28 days \\
Headache (grade 1) & 0 & & $3(5 \%)$ & 28 days \\
Headache (grade 2) & $2(4 \%)$ & 84 days & $1(2 \%)$ & 28 days \\
Sensitive headskin (grade 1) & $1(2 \%)$ & 14 days & $1(2 \%)$ & 56 days \\
Focal alopecia (grade 1) & $1(2 \%)$ & 21 days & 0 & \\
Concentration loss (grade 1) & $1(2 \%)$ & 56 days & $3(5 \%)$ & 28 days \\
Fatigue (grade 1) & $1(2 \%)$ & 9 days & 0 & \\
Dizziness (grade 1) & $1(2 \%)$ & 56 days & 0 & 1 day \\
Vertical diplopia (grade 1) & $1(2 \%)$ & 6 months & 0 & $3(5 \%)$ \\
Somnolence (grade 1) & $1(2 \%)$ & 18 days & 20 days & \\
Focal epilepsy (grade 1) & $1(2 \%)$ & & & \\
\hline
\end{tabular}

${ }^{\mathrm{a}}$ CTCAE (CTC-M) version 4.0 (common terminology criteria for adverse events) 


\section{Discussion}

In recent years, GKRS has become increasingly more popular as a salvage treatment modality for patients diagnosed with recurrent gliomas. The goal of GKRS for recurrent glioma patients is to improve survival rates with minimal burden for these patients. One can argue about what type of treatment is best for patients with recurrent glioma. This is dependent on several factors, such as patient's age, comorbidity, Karnofski-Index, histopathological diagnosis and available treatment modalities. In our center, a difference is made between low grade and high grade tumors. Patients with recurrent low grade tumors are more suitable to undergo reoperation because these tumors are less responsive to radiosurgery. However, this is dependent on location and tumor volume. Patients with recurrence in eloquent locations and/or with small tumor volume are less suitable to undergo reoperation and are more suitable candidates to undergo minimal invasive treatment such as GKRS. In case of high grade tumors, patients with recurrence have limited survival [12-14] and are more eligible for GKRS. Moreover, high grade tumors have a better radio-surgical response in comparison to low grade tumors. Finally, for patients with glioma the quality of life (QoL) is an increasingly important recognized factor. Not only do these patients suffer from general symptoms associated with cancer, such as fatigue, anxiety, and depression, but also from seizures, cognitive deficits, and focal neurological deficits. Therefore, treatments leading to deterioration of QoL are less favorable for these patients, giving their short lifespan, making GKRS an excellent treatment modality for maintaining QoL and extending survival time.

Determining radiological progression is known to be difficult for gliomas, especially after different treatment modalities have been applied. Until recently, clear definitions of progression and pseudo-progression have been lacking, which makes comparison between different studies on treating recurrent glioma bothersome. For this, the RANO (Response Assessment in Neuro-Oncology) criteria have been proposed to score radiological (pseudo-) progression more uniformally. However, these RANO criteria have not specifically been designed for patients treated with GKRS. Therefore, we have introduced the Tilburg radiological classification for scoring TC after GKRS. The primary goal of this classification is to allow us to compare our own treatment results more objectively. Furthermore, this classification could form the basis for uniform radiological criteria scoring radio-surgical response.

In our study, TC was achieved in $37 \%$ of all tumors; $50 \%$ in LGGs and $27 \%$ in HGGs. A recent study showed a $53.8 \%$ TC for LGGs after GKRS [15], which is comparable to our study. For HGGs, the TC is within range of other studies which reported TC of $18.8 \%-75.6 \%$ [16-19]. LP occurred in $46 \%$ of all tumors treated with GKRS. This might indicate a dose problem, meaning that a higher dose is needed to obtain a higher local TC. Another important subject is target definition which led to marginal progression in $9 \%$ of all tumors in our study. Koga et al. were the first to address these two problems [19]. They compared two groups, conventional SRS (20 Gy applied to the margin of each gadolinium-enhanced lesion) versus extended field SRS (margin was extended up to $0.5-1 \mathrm{~cm}$ ). $\mathrm{TC}$ in the conventional group was $47 \%$. By extending the margin of the clinical target volume the TC increased to 93\% $(\mathrm{P}=0.0035)$. However, there was no significant difference $(P=0.83)$ in survival time between the two groups. A higher incidence of radiation necrosis was seen in the extended SRS group (29\% vs. 6.5\%). The goal of extending the irradiation field is to include as many tumor cells invading the surrounding tissue as possible. A limitation of this method is that it is not applicable to large lesions. Extending the margin in a large target results in a large prescribed isodose volume and might cause uncontrollable radiation induced adverse events. In case of GKRS as salvage treatment, the goal is prolonged survival with minimal burden. Therefore we do not find it useful to irradiate more than the enhanced lesion because of the higher incidence of radiation necrosis and uncontrolled radiation induced adverse events. Gliomas are highly vascular tumors and have a tendency to infiltrate extensive areas of the brain. The real tumor may therefore be much larger than what is seen on MRI, making a $0.5-1 \mathrm{~cm}$ margin still insufficient. This is another reason to only irradiate the enhanced lesion for both high and low grade gliomas.

The median PFS and OS for all patients were 10.5 and 34.4 months, respectively. Median PFS was 50.1 months and 5.7 months for low and high grade tumors respectively. Median OS was 86.6 months and 12.8 months for low and high grade tumors respectively. This is comparable to other studies about GKRS for recurrent HGG who showed a median OS ranging from 13 to 14 months [18, 20-22].

Other studies in which radiosurgery was performed using a linear accelerator (LINAC) for the treatment of recurrent HGG showed comparable median OS ranging from 8.5 to 11 months [23-25]. As could be expected, there was a highly significant increase in both PFS and OS according to tumor grade (i.e. the lower the tumor grade, the better the outcome), reflecting the natural behaviour of these tumors and sensitivity to treatment. Of clinical interest, in recurrent GBM patients median OS was 10.4 months. This shows that GKRS can meaningfully lengthen survival in these patients with a 1 day hospitalization, maintenance of QoL and minimal burden. For patients with a limited life span, these advantages should make one consider GKRS as a treatment 
option in recurrent glioma patients, as opposed to systemic treatment modalities that might hamper QoL (and might increase the number of hospital visits needed for therapy).

It is important to note that there were no serious adverse events and that the therapy was well tolerated. In general, only mild adverse events were noticed and GKRS was well tolerated.

In the Netherlands, the first line of therapy for HGG is surgical resection followed by chemo-radiation [26]. In case of recurrence, several options do exist: repeat surgery, RT, chemotherapy, experimental therapy or a combination of these treatment modalities, all depending on the clinical state of the patient. We are one of only two centers in the Netherlands who have access to GKRS. In case of recurrent glioma we can use high single dose radiation for small (multiple) lesions without causing damage to surrounding brain tissue, which already has been exposed to radiation. This is a clear advantage over conventional RT. Therefore, our recommendation is to use GKRS if possible in small recurrent glioma since one can give a higher dose radiation in an already irradiated brain compared to conventional RT, minimizing the possible risk of side-effects.

We use triple dose gadolinium for the planning MRI-scan in combination with a small slice thickness of $1.5 \mathrm{~mm}$. This allows for detection of very small recurrence lesions. This is another advantage of using GKRS, because we can treat lesions in a very early stage of recurrence.

\section{Limitations of the study}

This is a single-center retrospective study with a relatively small number of patients and therefore subjected to biases (selection bias and treatment bias) and limitations. The recurrent glioma volume that was treated was rather small, as a result of selection bias. The patients that are eligible for GKRS treatment, are patients with smaller tumor volumes. As a result, the patients that are treated with GKRS probably have a less unfavourable prognosis. All patients were pre-treated before GKRS. Major limitations are nonprotocolized treatment regiments. The treatment included multiple surgeries, use of chemotherapeutic agents, different radiotherapy regimens, and heterogeneity in primary brain tumors. Therefore the group we present is very heterogeneous, which makes comparison to historical groups very difficult.

\section{Conclusion}

GKRS can safely be used as salvage treatment for recurrent glioma and seems to improve survival rates in (high grade) glioma patients with minimal burden.

\section{Compliance with ethical standards}

Conflict of interest The authors declare that they have no conflict of interest.

Open Access This article is distributed under the terms of the Creative Commons Attribution 4.0 International License (http://creativeco mmons.org/licenses/by/4.0/), which permits unrestricted use, distribution, and reproduction in any medium, provided you give appropriate credit to the original author(s) and the source, provide a link to the Creative Commons license, and indicate if changes were made.

\section{References}

1. Larjavaara S, Mantyla R, Salminen T, Haapasalo H, Raitanen J, Jaaskelainen J, Auvinen A (2007) Incidence of gliomas by anatomic location. Neuro-Oncology 9:319-325. https://doi. org/10.1215/15228517-2007-016

2. Gousias K, Markou M, Voulgaris S, Goussia A, Voulgari P, Bai M, Polyzoidis K, Kyritsis A, Alamanos Y (2009) Descriptive epidemiology of cerebral gliomas in northwest Greece and study of potential predisposing factors, 2005-2007. Neuroepidemiology 33:89-95. https://doi.org/10.1159/000222090

3. Ostrom QT, Gittleman H, Farah P, Ondracek A, Chen Y, Wolinsky Y, Stroup NE, Kruchko C, Barnholtz-Sloan JS (2013) CBTRUS statistical report: primary brain and central nervous system tumors diagnosed in the United States in 2006-2010. Neuro-Oncology 15 (Suppl 2):ii1-ii56 https://doi.org/10.1093/neuonc/not151

4. Crocetti E, Trama A, Stiller C, Caldarella A, Soffietti R, Jaal J, Weber DC, Ricardi U, Slowinski J, Brandes A (2012) Epidemiology of glial and non-glial brain tumours in Europe. Eur J Cancer 48:1532-1542 https://doi.org/10.1016/j.ejca.2011.12.013

5. Sant M, Minicozzi P, Lagorio S, Borge Johannesen T, MarcosGragera R, Francisci S (2012) Survival of European patients with central nervous system tumors. Int J Cancer 131:173-185. https:// doi.org/10.1002/ijc. 26335

6. Tseng MY, Tseng JH, Merchant E (2006) Comparison of effects of socioeconomic and geographic variations on survival for adults and children with glioma. J Neurosurg 105:297-305. https://doi. org/10.3171/ped.2006.105.4.297

7. Jung KW, Yoo H, Kong HJ, Won YJ, Park S, Lee SH (2012) Population-based survival data for brain tumors in Korea. J NeuroOncol 109:301-307. https://doi.org/10.1007/s11060-012-0893-5

8. Lee CH, Jung KW, Yoo H, Park S, Lee SH (2010) Epidemiology of primary brain and central nervous system tumors in Korea. J Korean Neurosurg Soc 48:145-152. https://doi.org/10.3340/ jkns.2010.48.2.145

9. Gigineishvili D, Shengelia N, Shalashvili G, Rohrmann S, Tsiskaridze A, Shakarishvili R (2013) Primary brain tumour epidemiology in Georgia: first-year results of a population-based study. J Neuro-Oncology 112:241-246. https://doi.org/10.1007/s1106 0-013-1054-1

10. Dubrow R, Darefsky AS (2011) Demographic variation in incidence of adult glioma by subtype, United States, 1992-2007. BMC Cancer 11:325. https://doi.org/10.1186/1471-2407-11-325

11. Cabrera AR, Kirkpatrick JP, Fiveash JB, Shih HA, Koay EJ, Lutz S, Petit J, Chao ST, Brown PD, Vogelbaum M, Reardon DA, Chakravarti A, Wen PY, Chang E (2016) Radiation therapy for glioblastoma: executive summary of an American society for radiation oncology evidence-based clinical practice guideline. Pract Radiat Oncol 6:217-225. https://doi.org/10.1016/j. prro.2016.03.007 
12. Biswas T, Okunieff P, Schell MC, Smudzin T, Pilcher WH, Bakos RS, Vates GE, Walter KA, Wensel A, Korones DN, Milano MT (2009) Stereotactic radiosurgery for glioblastoma: retrospective analysis. Radiat Oncol 4:11. https://doi. org/10.1186/1748-717x-4-11

13. Lamborn KR, Yung WK, Chang SM, Wen PY, Cloughesy TF, DeAngelis LM, Robins HI, Lieberman FS, Fine HA, Fink KL, Junck L, Abrey L, Gilbert MR, Mehta M, Kuhn JG, Aldape KD, Hibberts J, Peterson PM, Prados MD (2008) Progression-free survival: an important end point in evaluating therapy for recurrent high-grade gliomas. Neuro-Oncology 10:162-170. https://doi. org/10.1215/15228517-2007-062

14. Wong ET, Hess KR, Gleason MJ, Jaeckle KA, Kyritsis AP, Prados MD, Levin VA, Yung WK (1999) Outcomes and prognostic factors in recurrent glioma patients enrolled onto phase II clinical trials. J Clin Oncol 17:2572-2578. https://doi.org/10.1200/ jco.1999.17.8.2572

15. Gagliardi F, Bailo M, Spina A, Donofrio CA, Boari N, Franzin A, Fava A, Vecchio AD, Bolognesi A, Mortini P (2017) Gamma knife radiosurgery for low-grade gliomas: clinical results at longterm follow-up on tumor control and patients' quality of life. World Neurosurg. https://doi.org/10.1016/j.wneu.2017.02.041

16. Bokstein F, Blumenthal DT, Corn BW, Gez E, Matceyevsky D, Shtraus N, Ram Z, Kanner AA (2016) Stereotactic radiosurgery (SRS) in high-grade glioma: judicious selection of small target volumes improves results. J Neuro-Oncol 126:551-557. https:// doi.org/10.1007/s11060-015-1997-5

17. Skeie BS, Enger PO, Brogger J, Ganz JC, Thorsen F, Heggdal JI, Pedersen PH (2012) Gamma knife surgery versus reoperation for recurrent glioblastoma multiforme. World Neurosurg 78:658-669. https://doi.org/10.1016/j.wneu.2012.03.024

18. Elliott RE, Parker EC, Rush SC, Kalhorn SP, Moshel YA, Narayana A, Donahue B, Golfinos JG (2011) Efficacy of gamma knife radiosurgery for small-volume recurrent malignant gliomas after initial radical resection. World Neurosurg 76:128-140. https://doi. org/10.1016/j.wneu.2010.12.053 (discussion 161-122)

19. Koga T, Maruyama K, Tanaka M, Ino Y, Saito N, Nakagawa K, Shibahara J, Todo T (2012) Extended field stereotactic radiosurgery for recurrent glioblastoma. Cancer 118:4193-4200. https://doi.org/10.1002/cncr.27372

20. Kong DS, Lee JI, Park K, Kim JH, Lim DH, Nam DH (2008) Efficacy of stereotactic radiosurgery as a salvage treatment for recurrent malignant gliomas. Cancer 112:2046-2051. https://doi. org/10.1002/cncr.23402

21. Hsieh PC, Chandler JP, Bhangoo S, Panagiotopoulos K, Kalapurakal JA, Marymont MH, Cozzens JW, Levy RM, Salehi S (2005) Adjuvant gamma knife stereotactic radiosurgery at the time of tumor progression potentially improves survival for patients with glioblastoma multiforme. Neurosurgery 57:684-692 (discussion 684-692)

22. Szeifert GT, Prasad D, Kamyrio T, Steiner M, Steiner LE (2007) The role of the gamma knife in the management of cerebral astrocytomas. Prog Neurol Surg 20:150-163. https://doi. org/10.1159/000100102

23. Combs SE, Widmer V, Thilmann C, Hof H, Debus J, SchulzErtner D (2005) Stereotactic radiosurgery (SRS): treatment option for recurrent glioblastoma multiforme (GBM). Cancer 104:21682173. https://doi.org/10.1002/cncr.21429

24. Patel M, Siddiqui F, Jin JY, Mikkelsen T, Rosenblum M, Movsas B, Ryu S (2009) Salvage reirradiation for recurrent glioblastoma with radiosurgery: radiographic response and improved survival. J Neuro-Oncol 92:185-191. https://doi.org/10.1007/s1106 0-008-9752-9

25. Cho KH, Hall WA, Gerbi BJ, Higgins PD, McGuire WA, Clark HB (1999) Single dose versus fractionated stereotactic radiotherapy for recurrent high-grade gliomas. Int J Radiat Oncol Biol Phys 45:1133-1141

26. Stupp R, Mason WP, van den Bent MJ, Weller M, Fisher B, Taphoorn MJ, Belanger K, Brandes AA, Marosi C, Bogdahn U, Curschmann J, Janzer RC, Ludwin SK, Gorlia T, Allgeier A, Lacombe D, Cairncross JG, Eisenhauer E, Mirimanoff RO (2005) Radiotherapy plus concomitant and adjuvant temozolomide for glioblastoma. N Engl J Med 352:987-996. https://doi. org/10.1056/NEJMoa043330 